\title{
Canonically Transformed Detectors Applied to the Classical Inverse Scattering Problem
}

\author{
C JUNG, T H SELIGMAN and J M TORRES \\ Centro de Ciencias Físicas, Universidad Nacional Autónoma de México, Cuernavaca, \\ Morelos, México \\ E-mail: jung@fis.unam.mx,seligman@fis.unam.mx,mau@fisica.unam.mx
}

This article is part of the special issue published in honour of Francesco Calogero on the occasion of his 70th birthday

\begin{abstract}
The concept of measurement in classical scattering is interpreted as an overlap of a particle packet with some area in phase space that describes the detector. Considering that usually we record the passage of particles at some point in space, a common detector is described e.g. for one-dimensional systems as a narrow strip in phase space. We generalize this concept allowing this strip to be transformed by some, possibly nonlinear, canonical transformation, introducing thus a canonically transformed detector. We show such detectors to be useful in the context of the inverse scattering problem in situations where recently discovered scattering echoes could not be seen without their help. More relevant applications in quantum systems are suggested.
\end{abstract}

\section{Introduction}

Usually a classical scattering measurement will determine at what time the outgoing particle passes a certain point in a surface of codimension one in configuration space, which in one dimension is simply characterized by a distance from the center of the scattering region, and in more dimensions will also include scattering angles. If we allow for a non-zero thickness of the detector this will describe a region in configuration space. If in addition we consider that normally particles can be detected only within some finite energy range, this in turn implies a finite region in phase space. Depending on the characteristics of a real experiment or on theoretical advantages for a one-dimensional system it could be any structure narrow in configuration space and rather wider in momentum space. We now consider an experiment where the incoming particles form a narrow packet in phase space. The result of this experiment will be given as the time evolution of the overlap in phase space of the evolving packet with the phase space strip characterizing the detector.

The purpose of the present paper is to generalize this concept of a detector by allowing canonical transformations of the area in phase space that describes the detector, in order to be able to detect more subtle structures in the outgoing particle packet. We shall see that such generalized detectors are essential to solve the classical inverse scattering problem in 
certain cases, and we shall demonstrate the usefulness of the concept in a simple example, which will also provide typical transformations needed.

In two recent publications [1, 2] it has been suggested to use the self-pulsing effect of chaotic scattering ( scattering echoes ) to solve the chaotic inverse scattering problem. Recently an experiment in superconductive cavities [3] has confirmed the theoretical prediction [1, 2]. The basic idea is the following:

Imagine a scattering system with two essential degrees of freedom ( one of them may be a periodic explicit time dependence, the case we are mainly concerned with in the present paper ) which can be converted into a 2-dimensional iterated map, either a Poincaré map or a stroboscopic map. We consider the case where this map has a large KAM island surrounded by a homoclinic/heteroclinic tangle. Incoming particles form a narrow packet in phase space. Many of these particles undergo a direct scattering process and emerge immediately. The rest comes in contact with the homoclinic/heteroclinic tangle and rotates around the KAM island. After each complete rotation some leave the homoclinic tangle and produce an outgoing particle pulse. We call the sequence of outgoing pulses echoes. The time difference $\tau$ between adjacent echoes is determined by the time it takes to circle around the KAM island and this time in turn is given by the development stage of the homoclinic tangle. For example for the case of a symmetric ternary horseshoe, which we shall encounter later, this development is described by a parameter $\gamma$. It varies between zero for a separatrix line and one for a complete horseshoe [4] and relates to the rotation period $T$ as

$$
T=-2 \log _{3}(\gamma)+3 / 2
$$

Thereby in the end a measurement of the temporal distance between adjacent echoes gives the development degree of the homoclinic tangle.

The crucial point is a clean measurement of the echoes. If all particles in any echo would have exactly the same speed, then each packet would stay together and we could measure them at any distance from the target. However, in reality each echo consists of a group of particles with different speeds and therefore the packets have dispersion i.e. become broader with increasing distance from the target. At some distance the echoes become broader than the distance between two adjacent ones. A separation into a sequence of consecutive echoes is no longer possible. At first one could think of using a detector which only accepts a small velocity window. This might work if the velocity spread is not too large. However it becomes difficult because of two reasons. First, for attractive potentials, where the outer fixed point of the Poincaré map is located at infinity, some of the outgoing particles have velocities close to zero and then the relative spread of velocities is beyond any limit. Second, a small velocity window of the detector only accepts a small part of the particles and thereby leads to a very small signal strength. There are two completely different strategies to circumvent these problems. One possibility is a measurement of the energy dependence of the cross section and to Fourier transform from the energy domain to the time domain (see discussion in [2, 3]).

If we insist to measure the sequence of echoes directly the second possibility is a detector whose sensitivity depends on an appropriate combination of position and velocity, i.e. a detector whose sensitivity covers an appropriate strip in phase space or in the domain of the Poincaré map. The ideal case is to cover a single unstable tendril of the homoclinic tangle. This can be achieved by canonically transforming the phase space strip that describes 
the simple minded (inadequate) detector. We shall call this a canonically transformed detector (CTD). In one approach to the inverse scattering problem, discussed in [4, 5], a central point is to find to which hierarchical level a particular scattering trajectory (or the corresponding interval of continuity of scattering functions) belongs. In the present setup the answer to this question becomes almost trivial. If we place the set of initial conditions into a single stable tendril and the detector is sensitive in a single unstable tendril only, then the total delay time (measured in units of the period of the kick) is the hierarchical level up to an irrelevant global constant.

In the present paper we concentrate for simplicity on periodically kicked systems in a 1-dimensional position space where the homoclinic tangle extends to infinity. In the next section we shall discuss an example giving rise to a symmetric ternary horseshoe, and in the following section we shall show how a CTD can detect echoes that cannot be seen in the usual way. Some concluding remarks will complete our argument.

\section{The system and its homoclinic tangle}

We use the system described by the Hamiltonian

$$
H(q, p, t)=\frac{p^{2}}{2}+A V(q) \sum_{n=-\infty}^{\infty} \delta(t-n) .
$$

Here $q$ is the position and $p$ the momentum of the particle at time $t . V(q)=-\exp \left(-q^{2}\right)$ indicates the potential function of the kick and $A$ its strenght. It is a periodically kicked system on an one-dimensional configuration space with a purely attractive potential. We represent the dynamics by the stroboscopic map taken at times $t=n+1 / 2$, i.e. always in the middle between two consecutive kicks. This choice is adapted to the time reversal symmetry of our system in so far as time reversal corresponds to a reflection in the $q$ axis and this reflection maps stable manifolds into unstable manifolds and vice versa. The map is given in closed form as

$$
\begin{aligned}
& p_{n+1}=p_{n}-A V^{\prime}\left(q_{n}+p_{n} / 2\right) \\
& q_{n+1}=q_{n}+\frac{1}{2}\left(p_{n}+p_{n+1}\right)
\end{aligned}
$$

Because the potential is purely attractive the system does not have any point of no return in configuration space. Therefore recurrent trajectories are not confined to a finite part of configuration space and also the chaotic invariant set (homoclinic tangle) extends to infinity. The outermost fixed points of the horseshoe are located at infinity. Since in the asymptotic region the dynamics is invariant under translations in position this outer fixed point is parabolic. However, under inclusion of nonlinearities of the dynamics it has a stable and an unstable invariant manifold reaching inwards to the interaction region. The local branch of the stable manifold of the point at infinity consists of trajectories which run in position monotonically to infinity while the momentum converges to zero. The corresponding part of the unstable manifold is obtained by time reversal. The horseshoe is traced by the continuation of these invariant manifolds of the points at infinity. Figure 1 shows the stroboscopic map Eq. 2.2. In the center marked in green we see a few KAM tori around the central fixed point at the origin as well as a few secondary islands, all 


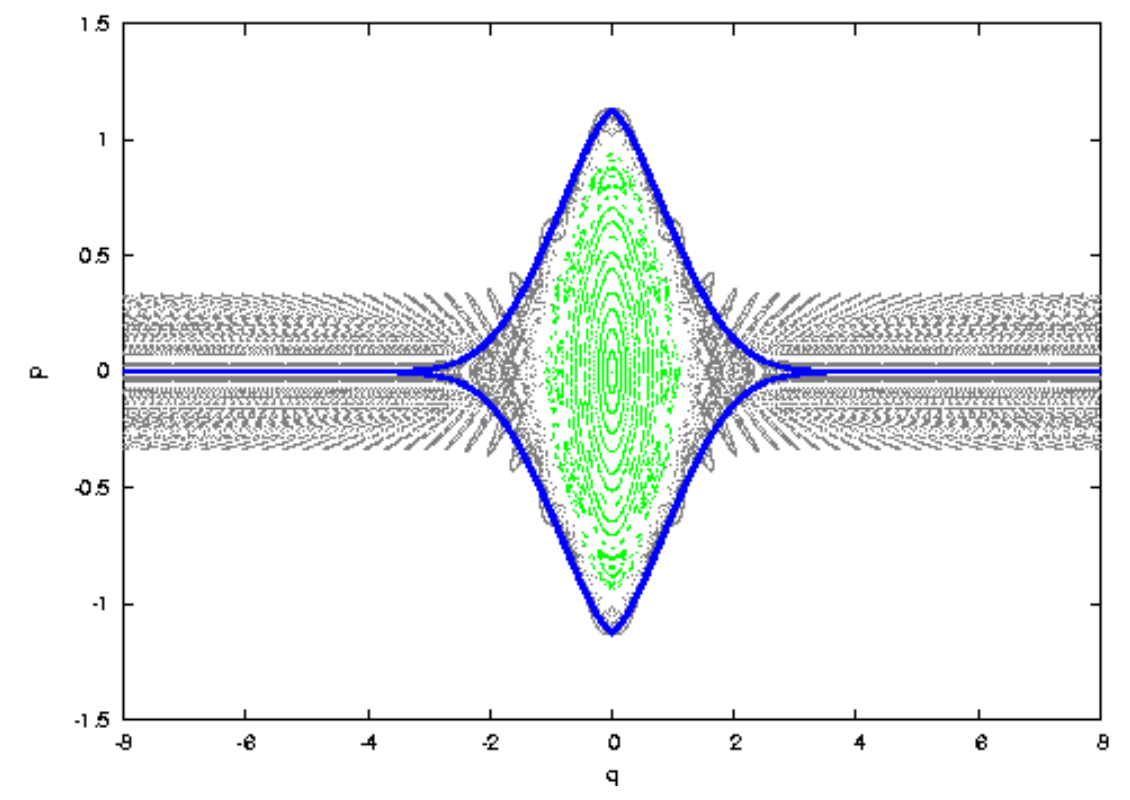

Figure 1. Horseshoe of low development in the stroboscopic map given by Eq. 2.2. KAM tori and secondary islands in green, the boundary of the fundamental rectangle $\mathrm{R}$ in blue and the tendrils of the invariant manifolds of the outer fixed points in grey.

inside the outermost KAM surface. In blue we mark the boundary of the fundamental rectangle $R$ [4, 6] that extends to infinity for zero momenta. The grey points are further iterations of the blue line and they show tendrils in the asymptotic region as well as in the chaotic layer between the outermost KAM surface and the boundary of $R$. This layer is interspersed with a fractal set of integrable islands, the largest of which appear as white areas within this layer. A small strip around the line of momentum zero in the domain of the map (its boundaries are the local segments of the invariant manifolds) belongs to the fundamental region $R$ of the horseshoe even for $q \rightarrow \pm \infty$. Therefore we exclude a larger strip from the asymptotic region and define the asymptotic region in phase space as the subset of the domain which, first, is far out in position space such that the potential is close to zero and, second, the absolute value of the momentum is large compared to the momentum values of the boundary of $R$. In the asymptotic region the dynamics converges to free motion and the action of the map converges to a pure shear motion. If we take as a set of initial conditions in the asymptotic region a circular spot in phase space and let it evolve under the asymptotic motion then it turns into a more and more elongated ellipse. The major axis turn more and more horizontal under further iteration. Figure 2 shows such an initial distribution in red and a few of its iterated images. In blue we see the evolution of a particle packet well defined in $q$ and with a fairly small spread in $p$ starting in the incoming asymptotic region. We see how it evolves emitting strongly deformed pulses in both directions that cover several tendrils. 

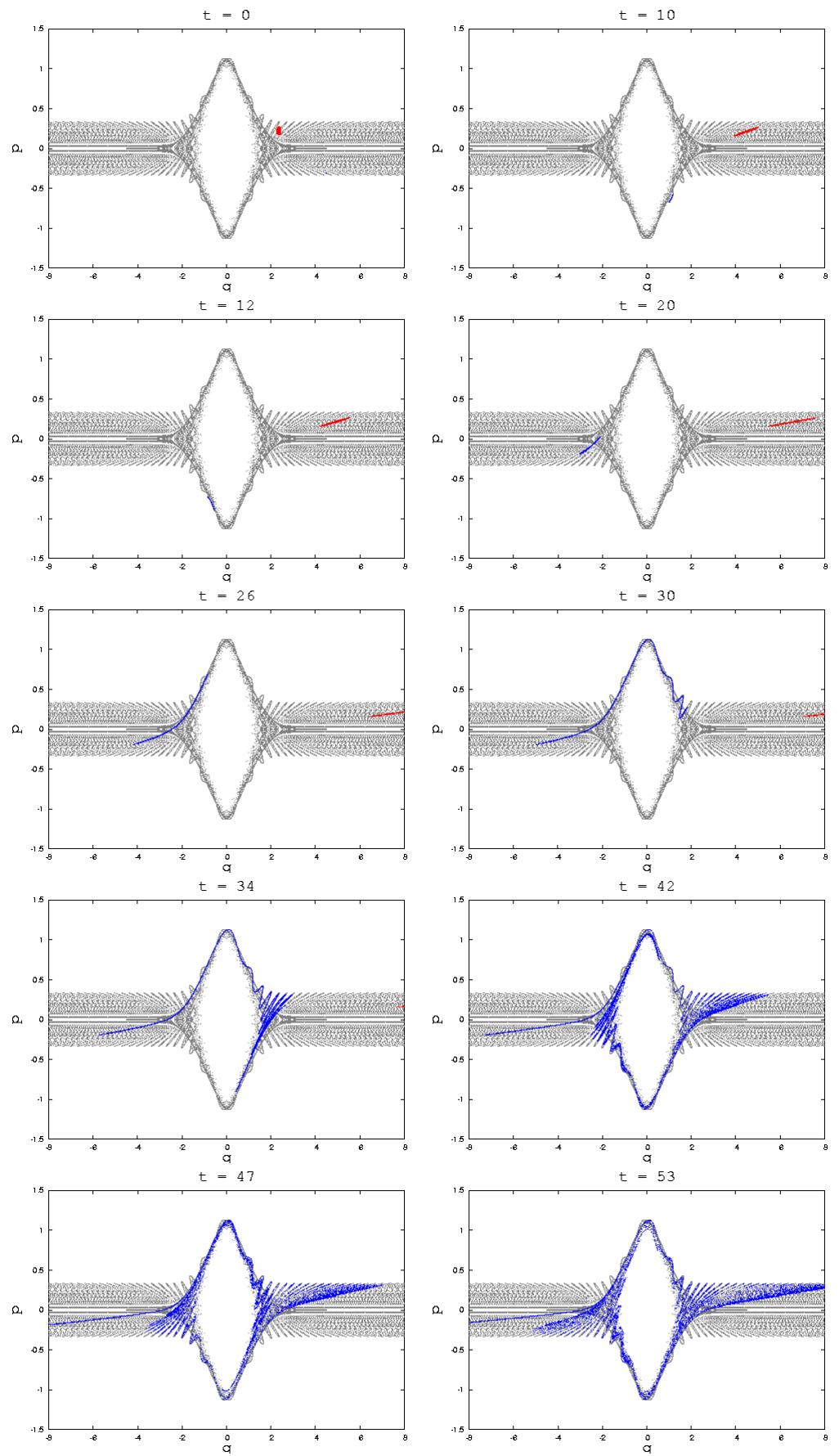

Figure 2. Superimposed on the horseshoe of Fig. 1 plotted in grey we show in red the time development of a small particle packet in the outgoing asymptotic region and in blue the time development of a small particle packet placed into the incoming asymptotic region. The various parts of the figure show the development at time values $0,10,12,20,26,30,34,42,47$ and 53 respectively. 


\section{Canonically transformed detectors}

Looking at Fig. 2 we suspect, that a detector described by a strip cutting through many tendrils in the outgoing region would not show any interesting features of the dynamics and certainly not resolve any echoes in the sense discussed in [1, 3. This would be the case even if the outgoing signal would start as a rather clean pulse due to the shear seen for the inicial conditions marked in red in Fig. 2] and certainly for the result of a scattering process as marked in blue in Fig. 2. Indeed Fig. 3a shows such a detector, and Fig. 3. shows the corresponding useless signal. The blue line gives the signal for a detector on the left-hand side, and the red line the one from a detector on the right-hand side, from which we sent the incoming pulse (c.f. Fig. 2).

We therefore search for a canonical transformation that transforms a line of some constant distance in configuration space, at which we would like to measure and cover an appropriate momentum interval, into a shape that adjusts to a single tendril. In the present case we choose

$$
\begin{aligned}
& P=p \\
& Q=q+\operatorname{sign}(q) f(p)
\end{aligned}
$$

where

$$
f(p)=(7|p|)^{1.3}+16|p|-4.6
$$

The transformation only makes sense in the asymptotic region and is only used there. The corresponding detector is depicted in Fig. 3 $\mathrm{b}$, and in Fig. 3 $\mathrm{d}$ we can now clearly detect three echoes. As expected the echoes to the right and to the left are in counter phase.

We thus clearly see that the CTD produced a useful signal, where the original detector did not. Yet we may ask, why we do not see signals as neat and numerous as in ref. [2]. There the incoming particle pulse was launched around the outer branch of the stable manifold of the fixed point. This branch does not exist in the present case and a launch near the inner branch of the unstable manifold does not make much sense due to the very low momenta. Yet we could use the same procedure as for the detector and create an incoming packet that would mimic the shape of a tendril and furthermore be sufficiently

narrow to lie within one or a few intervals of continuity. This would obviously produce the desired neat image. Yet having a canonically transformed initial packet and detector is fortunately more then we really need to see the echoes. But we should keep in mind, that we can operate on the pulses or on the detectors.

\section{Discussion and final remarks}

We have seen, that CTD's, as obtained by canonically transforming more conventional detectors are very helpful to produce scattering data, that allow to understand the inverse scattering problem.

As an example we used a periodically kicked system in a one-dimensional position space. Generalization to any scattering system with two important degrees of freedom where one of them is open and the other one closed result trivial [5]. In truely two-dimensional 

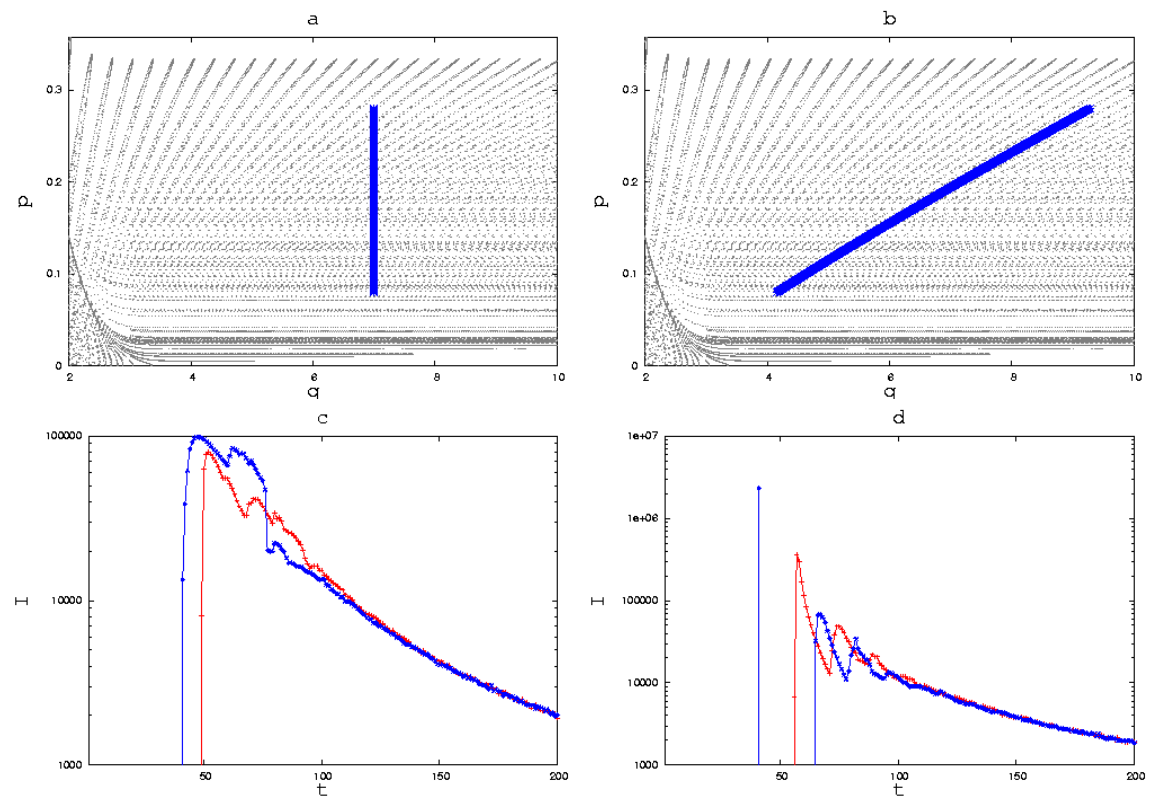

Figure 3. The "standard" detector (a) and the canonically transformed detector (b) are depicted together with the corresponding number of particle counts $I$ on a semi-log scale as a function of time $t$ in (c) and (d). The counts are integrated over one period of the map and shown in blue for a detector on the left and in red for a dector on the right. The lines are drawn to guide the eye. Note the echoes in counterphase in (d)

systems we have to construct an appropriate Poincaré section that allows the relevant horseshoe construction [4, 6]

Here we must recall that probably the main applications of the classical inverse scattering problem arise in the context of semi-classical behaviour of wave and quantum systems, as best exemplified by an experiment devised explicitly for this goal 3. Apart from this specially designed experiment several practical situations occur. Examples are: The collinear scattering of a particle from a two particle bound state where collisional excitation of the vibration occurs; a system with any periodic drive by an external field (laser assisted collisions); the motion of an electron in a channel e.t.c.. In [5] it has become evident that we need a detector which measures position and momentum simultaneously to extract information for the inverse problem in the case of collisional excitations of the closed degree of freedom, but from the considerations in the present paper it is also clear, that the shear in outgoing asymtotic motion will make canonically transformed detectors necessary even in systems that display echoes in the near field as soon as we move the detectors sufficiently far away.

Yet the most important perspective is the option to realize this idea in quantum optics, where overlaps with coherent states are commonly considered as measurements [7], and overlap with squeezed states is an obvious extension; yet the posibility of using canonically transformed coherent states in a more general setting opens new perspectives, which we plan to explore in a future paper. 


\section{Acknowledgments}

The authors acknowledge helpful discussions with C. Mejia-Monasterio, W. Schleich and V. Manko as well as financial support by DGAPA project IN-101603 and CONACyT project $43375-\mathrm{F}$.

\section{References}

[1] C. Jung, C. Mejia-Monasterio and T. H. Seligman, Europhys. Lett. 55, 616 (2001)

[2] C. Jung, C. Mejia-Monasterio, O. Merlo and T. H. Seligman, New J. Phys. 6, 48 (2004)

[3] C. Dembovsky et. al. Phys. Rev. Lett (2004) (in press) (nlin.CD/0408046)

[4] C. Jung, C. Lipp and T. H. Seligman, Ann. Phys. 275, 151 (1999)

[5] H. Tapia and C. Jung, Phys. Lett. A 313, 198 (2003)

[6] C. Jung and H.J. Scholz, J. Phys. A, 20, 3607 (1987)

[7] W. Schleich, Quantum Optics in Phase Space, Wiley-VCH, Weinheim, 2001 